\title{
Diffuse-interface simulations of drop coalescence and retraction in viscoelastic fluids
}

\author{
Pengtao Yue ${ }^{\mathrm{a}}$, James J. Feng ${ }^{\mathrm{a}, *}$, Chun Liu ${ }^{\mathrm{b}}$, Jie Shen ${ }^{\mathrm{c}}$ \\ ${ }^{a}$ Department of Chemical and Biological Engineering and Department of Mathematics, \\ University of British Columbia, Vancouver, Canada BC V6T $1 Z 4$ \\ b Department of Mathematics, The Pennsylvania State University, University Park, PA 16802, USA \\ ${ }^{\mathrm{c}}$ Department of Mathematics, Purdue University, West Lafayette, IN 47907, USA
}

Received 8 December 2004; received in revised form 30 April 2005; accepted 11 July 2005

\begin{abstract}
Drop dynamics plays a central role in defining the interfacial morphology in two-phase complex fluids such as emulsions and polymer blends. In such materials, the components are often microstructured complex fluids themselves. To model and simulate drop behavior in such systems, one has to deal with the dual complexity of non-Newtonian rheology and evolving interfaces. Recently, we developed a diffuse-interface formulation which incorporates complex rheology and interfacial dynamics in a unified framework. This paper uses a twodimensional implementation of the method to simulate drop coalescence after head-on collision and drop retraction from an elongated initial shape in a quiescent matrix. One of the two phases is a viscoelastic fluid modeled by an Oldroyd-B equation and the other is Newtonian. For the parameter values examined here, numerical results show that after drop collision, film drainage is enhanced when either phase is viscoelastic and drop coalescence happens more readily than in a comparable Newtonian system. The last stage of coalescence is dominated by a short-range molecular force in the model that is comparable to van der Waals force. The retraction of drops from an initial state of zero-velocity and zero-stress is hastened at first, but later resisted by viscoelasticity in either component. When retracting from an initial state with pre-existing stress, produced by cessation of steady shearing, viscoelasticity in the matrix hinders retraction from the beginning while that in the drop initially enhances retraction but later resists it. These results and the physical mechanisms that they reveal are consistent with prior experimental observations.
\end{abstract}

(C) 2005 Elsevier B.V. All rights reserved.

Keywords: Phase-field method; Interfacial dynamics; Film drainage; van der Waals force; Disjoining pressure; Dynamic interfacial tension

\section{Introduction}

Drop dynamics is the key to understanding interfacial morphology in two-phase materials[1]. In nature and in industrial processes, many such materials have components that are complex fluids themselves, with internal microstructures whose evolution affects the macroscopic dynamics of the material, especially the rheology. Examples include polymer blends [2], polymer-dispersed liquid crystals [3] and various biological fluids [4].

Theoretical and numerical analysis of drop dynamics in complex fluids has to struggle with the dual difficulties of

\footnotetext{
* Corresponding author.

E-mail address: jfeng@chml.ubc.ca (J.J. Feng).
}

moving interfaces and microstructure-dependent rheology. Specifically, there is the interplay among microscopic, mesoscopic and macroscopic scales: (a) the internal microstructure, e.g., molecular conformation, inside each component; (b) the interfaces and (c) the flow field. The coupling between (b) and (c) alone is well studied for Newtonian drops [5]. Similarly, the (a)-(c) coupling is the subject of molecular constitutive theories in rheology (e.g., [6]). Having both (a) and (b) present in a flow problem is the novelty of this work.

Methods for solving moving-interface problems fall into two broad categories: interface tracking and interface capturing $[7,8]$. The former uses a moving mesh with grid points residing on the interface. The latter determines the position of the interface by using a scalar function, whose evolution is typically represented by an advection equation on a fixed 
grid. Conceptually, both treat the interface as a zero-thickness surface, though an essential ingredient in the fixed-grid methods is a numerical regularization that spreads the interfacial force over a volume.

Recently, Yue et al. [9] proposed a fixed-grid diffuseinterface model for two-phase flows of complex fluids. This model differs from other fixed-grid methods in that the interface is treated as physically diffuse (e.g., $[10,11])$. The interfacial position and thickness are determined by a phase-field variable whose evolution is governed by a mixing energy. This way, the structure of the interface is rooted in molecular forces; the tendencies for mixing and demixing are balanced through the nonlocal mixing energy. This contrasts the level set and volume-of-fluid methods, which replace the surface tension by a body force or stress as a numerical device to regularize the singularity. The significance of this physical root will become apparent when we discuss the rupture of the thin film separating two coalescing drops. When the interfacial width approaches zero, the diffuse-interface model becomes identical to a sharp-interface level-set formulation. It also reduces properly to the classical sharp-interface model.

In our context of two-phase complex fluids, another attraction of the diffuse-interface method is its capability of easily incorporating the rheology of microstructured fluids. This is by virtue of its energy-based variational formalism. As long as the conformation of the microstructure is describable by a free energy, this energy can be added to the mixing energy to form the total free energy of the multi-phase system. Then a formal variational procedure applied to the total free energy will give rise to the proper constitutive equation for the microstructured fluids in addition to the evolution equation of the phase field variable. Using the Frank distortion energy for a liquid crystal, Yue et al. [9] illustrated how interfacial dynamics and complex rheology can be included in a unified theoretical framework. Dissipative effects such as viscous stresses, of course, have to be accounted for separately, e.g., via the standard irreversible thermodynamic procedure [12] or by including Brownian motion in Hamilton's principle $[13,14]$.

Yue et al. [9] have implemented the diffuse-interface method using a spectral representation, and presented preliminary numerical results to validate the theoretical model and the numerical method. The goal of this paper is to apply the method to physically interesting problems where it generates new insights into the physics. We will investigate two problems: drop coalescence after head-on collision and drop retraction from an elongated initial shape. The far-field matrix fluid remains quiescent in both problems.

\section{Theory and numerical method}

Yue et al. [9] have given a detailed derivation of the theoretical model, discussed its strengths and weaknesses and its relationship with other fixed-grid methods, and described the numerical scheme using spectral discretization. In this section, we will specialize the formalism for a mixture of a Newtonian and an Oldroyd-B fluid, and summarize the main features of the numerical procedure.

The Newtonian and Oldroyd-B components are immiscible except in a very thin interfacial region. This diffuse interface has a small but non-zero thickness, inside which the two components are mixed and store a mixing energy. An Oldroyd-B fluid consists in a dilute suspension of linear Hookean dumbbells in a Newtonian solvent [15]. The total free energy of the mixture thus comprises two parts: mixing energy of the interface and elastic energy for the dumbbells.

We introduce a phase-field variable $\phi$ such that the concentrations of the Oldroyd-B and Newtonian components are $(1+\phi) / 2$ and $(1-\phi) / 2$, respectively. For the mixing energy, we adopt the familiar Ginzburg-Landau form:

$f_{\text {mix }}(\phi, \nabla \phi)=\frac{1}{2} \lambda|\nabla \phi|^{2}+\frac{\lambda}{4 \epsilon^{2}}\left(\phi^{2}-1\right)^{2}$,

where $\lambda$ is the mixing energy density with the dimension of force, and $\epsilon$ is a capillary width that scales with the thickness of the diffuse interface. As $\epsilon \rightarrow 0$, the ratio $\lambda / \epsilon$ produces the interfacial tension in the classical sense [16,9]. The evolution of $\phi$ is governed by the Cahn-Hilliard equation:

$$
\frac{\partial \phi}{\partial t}+\boldsymbol{v} \cdot \nabla \phi=\gamma \lambda \nabla^{2}\left[-\nabla^{2} \phi+\frac{\phi\left(\phi^{2}-1\right)}{\epsilon^{2}}\right]
$$

where $\gamma \lambda$ determines the relaxation time of the interfacial profile [9].

For a single dumbbell with a connector $Q$, its elastic energy is $\frac{1}{2} H \boldsymbol{Q} \cdot \boldsymbol{Q}$, where $H$ is the elastic constant. For an ensemble of dumbbells with configuration distribution $\Psi(\boldsymbol{Q})$, the average energy can be written as

$f_{\mathrm{d}}=\int_{R^{3}}\left(k T \ln \Psi+\frac{1}{2} H \boldsymbol{Q} \cdot \boldsymbol{Q}\right) \Psi \mathrm{d} Q$,

where $k$ is the Boltzmann constant and $T$ is the temperature, and the integration is over all possible configurations of $\boldsymbol{Q}$. Now the total free energy density of the two-phase system is:

$f=f_{\text {mix }}+\frac{1+\phi}{2} n f_{\mathrm{d}}$,

where $n$ is the number density of the dumbbells.

A variational procedure applied to the total free energy will yield the elastic stress tensor for the system. The stress tensor due to $f_{\text {mix }}$ has been derived by Yue et al. [9], and here we will only consider the elastic stress due to the dumbbell energy $f_{\mathrm{d}}$. We impose a virtual displacement $\delta \boldsymbol{x}$ on the material, which takes place instantaneously so that the dumbbells deform affinely with no slip between the bead and the surrounding fluid:

$\delta \boldsymbol{Q}=\boldsymbol{Q} \cdot \nabla \delta \boldsymbol{x}$. 
The corresponding change in the distribution function $\Psi$ can be calculated from the continuity of $\Psi$ [15]:

$\delta \Psi=-\frac{\partial}{\partial \boldsymbol{Q}} \cdot(\delta \boldsymbol{Q} \Psi)$.

This in turn causes a variation in the dumbbell free energy:

$$
\begin{aligned}
\delta f_{\mathrm{d}}= & \int_{R^{3}}\left(k T \ln \Psi+k T+\frac{H}{2} \boldsymbol{Q} \cdot \boldsymbol{Q}\right) \delta \Psi \mathrm{d} Q \\
= & \int_{R^{3}}\left(k T \ln \Psi+\frac{H}{2} \boldsymbol{Q} \cdot \boldsymbol{Q}\right)\left[-\frac{\partial}{\partial \boldsymbol{Q}} \cdot(\boldsymbol{Q} \cdot \nabla \delta \boldsymbol{x} \Psi)\right] \\
& \times \mathrm{d} Q=\int_{R^{3}}\left(k T \boldsymbol{Q} \frac{\partial \Psi}{\partial \boldsymbol{Q}}+H \boldsymbol{Q} \boldsymbol{Q} \Psi\right) \mathrm{d} Q:(\nabla \delta \boldsymbol{x})^{\mathrm{T}} \\
= & \int_{R^{3}}\left[-k T\left(\frac{\partial \boldsymbol{Q}}{\partial \boldsymbol{Q}}\right)^{\mathrm{T}}+H \boldsymbol{Q} \boldsymbol{Q}\right] \Psi \mathrm{d} Q:(\nabla \delta \boldsymbol{x})^{\mathrm{T}} \\
= & (-k T \boldsymbol{I}+H\langle\boldsymbol{Q} \boldsymbol{Q}\rangle):(\nabla \delta \boldsymbol{x})^{\mathrm{T}},
\end{aligned}
$$

where $\langle\cdot\rangle=\int_{R^{3}} \cdot \Psi \mathrm{d} Q$ and $\boldsymbol{I}$ is the identity tensor. Thus, the dumbbell stress tensor is:

$\boldsymbol{\tau}_{\mathrm{d}}=-n k T \boldsymbol{I}+n H\langle\boldsymbol{Q} \boldsymbol{Q}\rangle$

This is exactly the Kramers expression for the polymer elastic stress tensor [15], and $\boldsymbol{\tau}_{\mathrm{d}}$ obeys the Maxwell equation:

$\boldsymbol{\tau}_{\mathrm{d}}+\lambda_{\mathrm{H}} \boldsymbol{\tau}_{\mathrm{d}(1)}=\mu_{\mathrm{p}}\left[\nabla \boldsymbol{v}+(\nabla \boldsymbol{v})^{T}\right]$,

where the subscript (1) denotes the upper convected derivative, $\lambda_{\mathrm{H}}=\zeta /(4 H)$ is the relaxation time, $\zeta$ being the friction coefficient between the dumbbell beads and the suspending solvent, and $\mu_{\mathrm{p}}=n k T \lambda_{\mathrm{H}}$ is the polymer viscosity. Adding the interfacial elastic stress [9] and the proper viscous stress, we obtain the total stress tensor:

$$
\begin{aligned}
\boldsymbol{\tau}= & \left(\frac{1-\phi}{2} \mu_{\mathrm{n}}+\frac{1+\phi}{2} \mu_{\mathrm{s}}\right)\left[\nabla \boldsymbol{v}+(\nabla \boldsymbol{v})^{T}\right]+\frac{1+\phi}{2} \boldsymbol{\tau}_{\mathrm{d}} \\
& +\lambda \nabla \phi \nabla \phi,
\end{aligned}
$$

where $\mu_{\mathrm{n}}$ is the viscosity of Newtonian component and $\mu_{\mathrm{s}}$ is the viscosity of the Newtonian solvent.

Eqs. (2) (9) and (10), along with the continuity and momentum equations $\nabla \cdot \boldsymbol{v}=0$ and $\rho\left(\frac{\partial v}{\partial t}+\boldsymbol{v} \cdot \nabla \boldsymbol{v}\right)=-\nabla p+$ $\nabla \cdot \tau$, form the governing equations for our two-phase system.

We use a two-dimensional (2D) Fourier spectral method for solving these equations, and an interested reader can find details of the numerical algorithm in [9,17]. All simulations are done on a regular mesh in a rectangular domain with periodic boundary conditions in both directions. To enhance stability, we advance time semi-implicitly, with the non-linear transport terms treated explicitly while the linear terms implicitly. The number of operations per time step scales as $O(N \log N), N$ being the number of unknowns. We have carried out mesh and time-step refinements to determine the adequacy of our spatial and temporal resolutions. The interface typically requires $7-10$ grids to resolve, and the total number of grid points is typically $2048 \times 1024$. For the time step, we find the Courant-Friedricks-Lewy condition a useful guideline. In all cases tested, the temporal resolution is adequate as long as the simulation is stable.

The two-dimensionality puts a considerable limitation on our work, and precludes quantitative comparison with experiments. Since the curvature in the third dimension is not accounted for, drop relaxation tends to occur at a different speed than in reality, for example. Our spectral method uses a regular mesh, and adequate resolution of the interface in three dimensions will be prohibitively expensive. Work is under way to develop an adaptive meshing scheme within a finite-element framework in three dimensions.

\section{Drop coalescence after head-on collision}

An advantage of the diffuse-interface method is its ability to handle topological changes in the interfacial morphology, and this section exploits this ability to probe the coalescence of two drops after a head-on collision. Yue et al. [9] illustrated this ability by a single example of Newtonian drops coalescing in a Newtonian matrix. Here we explore two physically important issues: (a) the representation of short-range molecular forces in the diffuse-interface framework; (b) the effects of viscoelasticity in either component on the collision and coalescence.

We have adopted the scheme of Nobari et al. [18] to simulate the head-on collision of two identical drops. Initially, two stationary drops of diameter $D$ are separated by a center-tocenter distance $d$. Then a body force $f$ is applied to accelerate the drops toward each other. When the drop velocity attains a prescribed value $U / 2, \boldsymbol{f}$ is turned off. The two drops collide into each other by inertia and coalesce.

\subsection{Newtonian drops in a Newtonian matrix: molecular forces}

Fig. 1 illustrates a typical run for two Newtonian drops in a Newtonian matrix of the same viscosity. The initial separation between the centers of the drops is $d=1.5 \mathrm{D}$, and the body force is turned on at $t=0$ and off at $t=0.336$ to achieve a $U$ value that corresponds to $R e=33.6$. Time is scaled here by $D / U$. The drops deform while approaching each other $(t=1.342)$. As a result, a thin film of the matrix fluid forms between the two drops. The thinning of this film, via the drainage of the matrix fluid through the narrowing conduit, determines the time scale of coalescence. The drainage requires a high pressure in the middle of the film, which produces a "dimpled shape" for the interface [19], with the minimum film thickness not at the middle point but farther out toward the sides. Rupture of the film at those locations traps a filament of the matrix fluid inside the resultant large drop. The subsequent breakup of the filament into drops is not due to capillary instability; rather it is caused by the stretching flow due to the bulging interface [9]. The most important feature, however, is that coalescence 

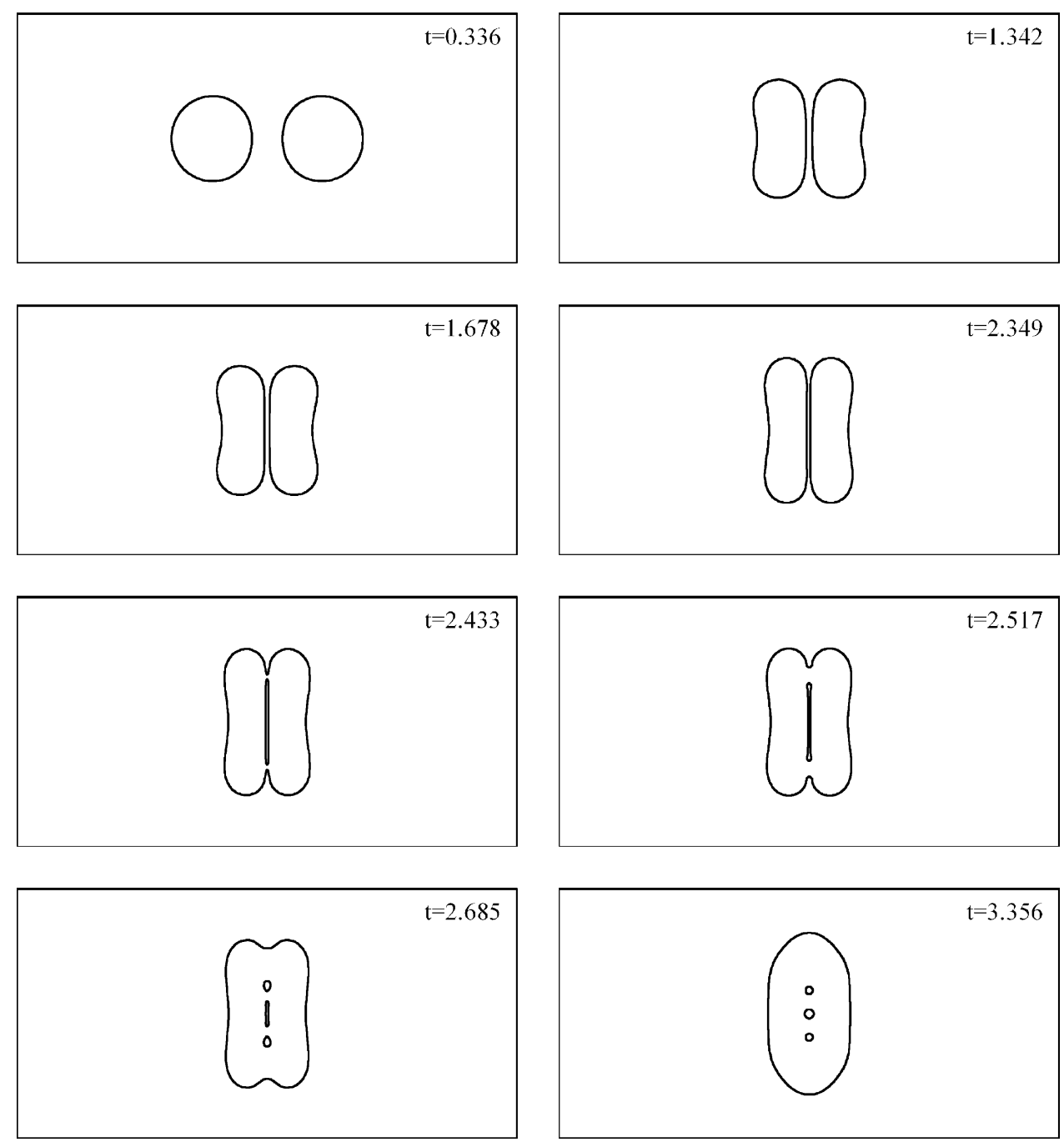

Fig. 1. Collision and coalescence of two Newtonian drops in a Newtonian matrix. The Reynolds number, defined using $D$ and $U$, is $R e=33.6$, and the Weber number is $W e=12$. Other parameters are: $\epsilon=0.01$ and $\gamma=3.365 \times 10^{-5}$ (after Yue et al. [9], @Cambridge University Press.).

occurs "naturally" as a result of the Cahn-Hilliard dynamics (cf. Eq. (2)). This contrasts sharp-interface simulations where the film separating the two drops has to be artificially removed to bring about the topological change [18].

A natural question is whether the breakage and reconnection of the interfaces simulated here reflects reality. According to our current understanding, the rupture of an interface is not a purely hydrodynamic process. As the film gets to a critical thickness of tens of nanometers, van der Waals attraction overcomes electric double-layer repulsion and causes the film to rupture and the two interfaces to merge [20-22]. This type of molecular interaction is what the Cahn-Hilliard mixing energy strives to represent. In this sense, the diffuse-interface model contains the physics of short-range molecular forces. However, the form of the Cahn-Hilliard force turns out to be different from the familiar van der Waals force.

Consider a liquid film (F) of uniform thickness $h$ sandwiched between semi-infinite domains of another fluid (A) (Fig. 2). The van der Waals interaction energy per unit area between the parallel interface can be written as [23]:

$$
E_{\mathrm{A}}=-\frac{A_{h}}{12 \pi h^{2}},
$$

where $A_{h}$ is the Hamaker constant. The intervening film would in general hamper the van der Waals interaction, but we neglect this effect here for simplicity. From this energy we can get the disjoining pressure

$\Pi \equiv p_{\mathrm{A}}-p_{\mathrm{F}}=-\frac{\mathrm{d} E_{\mathrm{A}}}{\mathrm{d} h}=-\frac{A_{h}}{6 \pi h^{3}}$.

To derive the counterpart of the disjoining pressure in the diffuse-interface representation, we consider the equilibrium $\phi(y)$ profile in the configuration of Fig. 2. In the bulk phase $\mathrm{A}, \phi \rightarrow 1$. If the film is much thicker than the interfaces $(h \gg \epsilon)$, then $\phi(0)$ would approach -1 in the film phase. Since the film is thin, however, $\phi(0)=\phi_{0}>-1$. Now the disjoining pressure can be calculated from the stress field. Note that the mixing energy of Eq. (1) produces an elastic stress tensor [9]: 


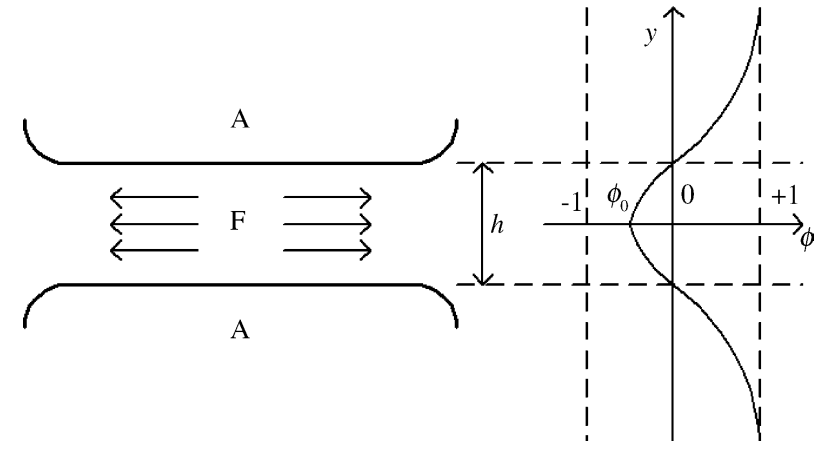

Fig. 2. A draining film and the corresponding $\phi$ profile.

$\boldsymbol{T}=-\left(p-f_{\text {mix }}\right) \boldsymbol{I}-\lambda \nabla \phi \nabla \phi$,

where $p$ is the thermodynamic pressure, and $\boldsymbol{I}$ is the secondorder unit tensor. In the bulk phase A, the normal stress in the $y$ direction is simply $-p_{\mathrm{A}}$. At the center of the film, $\left.\frac{\mathrm{d} \phi}{\mathrm{d} y}\right|_{y=0}=0$ because of symmetry. Thus, the normal stress there is $-p_{\mathrm{F}}+\lambda f_{0}$, where $f_{0}=\left(\phi_{0}^{2}-1\right)^{2} / 4 \epsilon^{2}$ is the local part of the mixing energy. A normal force balance then gives the disjoining pressure in the diffuse-interface model as

$\Pi_{\phi}=-\lambda f_{0}=-\frac{\lambda\left(\phi_{0}^{2}-1\right)^{2}}{4 \epsilon^{2}}$.

The disjoining pressure in Eq. (14) has the following in common with the van der Waals force in Eq. (12):

- The disjoining pressure is negative, indicating attraction between the two interfaces;

- The attraction is a short-distance force. It becomes significant only if the film is sufficiently thin $(h \sim \epsilon)$; for large $h$, both $\Pi$ and $\Pi_{\phi}$ vanish.

There are notable differences, however. As $h \rightarrow 0$, the van der Waals force diverges: $\Pi \rightarrow \infty$. On the other hand, as the two diffuse interfaces merge, $\phi_{0} \rightarrow 0$ and $\Pi_{\phi}$ approaches a finite value $-\lambda / 4 \epsilon^{2}$. For a film of thickness $h \sim \epsilon$, the two expressions for the disjoining pressure have the same order of magnitude. This can be seen by noting the connection between the Hamaker constant and the surface tension [23]:

$\sigma=\frac{\sqrt{3}}{2 \pi^{2}} \frac{A_{h}}{\xi^{2}}$,

where $\xi$ is the intermolecular center-to-center distance. Noting that $\sigma=(2 \sqrt{2} / 3)(\lambda / \epsilon)[9]$, we transform Eq. (12) into

$\Pi=-\frac{2 \sqrt{2} \pi}{9 \sqrt{3}} \frac{\lambda}{\epsilon^{2}}\left(\frac{\xi}{\epsilon}\right)^{2}\left(\frac{\epsilon}{h}\right)^{3}$,

which, for $h \sim \epsilon \sim \xi$, is on the same order as $\Pi_{\phi}$ in Eq. (14). To estimate $\Pi_{\phi}$ for a non-vanishing $h$, we may assume a hyperbolic tangent $\phi$-profile as in a one-dimensional equilibrium interface [9]. Then the phase-field function in the middle of the film can be estimated:

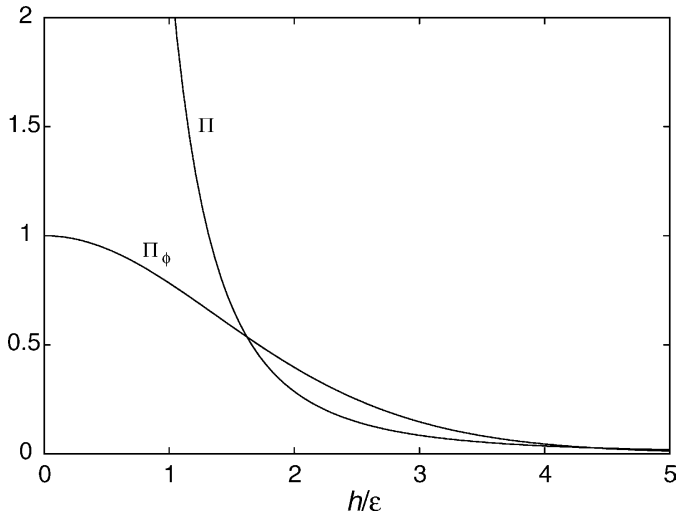

Fig. 3. Comparison of the disjoining pressure $\Pi_{\phi}$ in the diffuse-interface model, computed from Eqs. (14) and (17), with $\Pi$ of Eq. (16) derived from the van der Waals force. The two pressures are scaled by $-\lambda / 4 \epsilon^{2}$, and we have assumed $\epsilon=\xi$ in Eq. (16).

$\phi_{0}=-\tanh \left(\frac{h}{2 \sqrt{2} \epsilon}\right)$.

Inserting this into Eq. (14), we illustrate the quantitative differences between $\Pi_{\phi}$ and $\Pi$ at different $h$ values in Fig. 3 . On a fundamental level, the discrepancy between $\Pi$ and $\Pi_{\phi}$ stems from the truncation of the Cahn-Hilliard free energy at the quadratic term $|\nabla \phi|^{2}$. An elegant explanation has been given by Pismen [24]. Finally, one must note that the CahnHilliard dynamics is phenomenological in nature, and alternative diffusion mechanisms are available [25].

Besides the disjoining pressure, two additional factors affect the film drainage and rupture in a diffuse-interface framework. The first is the capillary thickness $\epsilon$, which can be seen as a measure of the thickness of the interface. Figs. 4 and 5 show simulations with a larger or smaller $\epsilon$ than in Fig. 1 with all other parameters unchanged. The early stage of the simulations, say for $t \leq 1.342$, is identical with Fig. 1. This is before the interfacial profiles of the two drops overlap. For a larger $\epsilon$, the interfaces of the two drops overlap at an earlier time during their approach, and the ensuing coalescence occurs more readily (Fig. 4). Note that the interface does not have time to develop the dimpled shape, and no matrix fluid is trapped inside the drop. On the other hand, a smaller $\epsilon$ prolongs the coalescence process (Fig. 5). As compared with Fig. 1, the points of rupture are more toward the ends of the film. This produces a less pronounced waist in the resultant compound drop, and the entrapped matrix filament does not break up but retracts into a droplet. The effect of $\epsilon$ is not to be confused with numerical resolution of the interface. For each $\epsilon$, mesh refinement has confirmed that the grid used here is adequate for resolving the interface (see also [9]). The optimal $\epsilon$ cannot be determined by an a priori criterion. Rather, it needs to reflect the range of the molecular forces at work in the particular experiment to be simulated.

The coalescence process also depends on the global curvature of the interfaces. In other words, it depends on how deformed the drops are at the start of the film drainage process. 

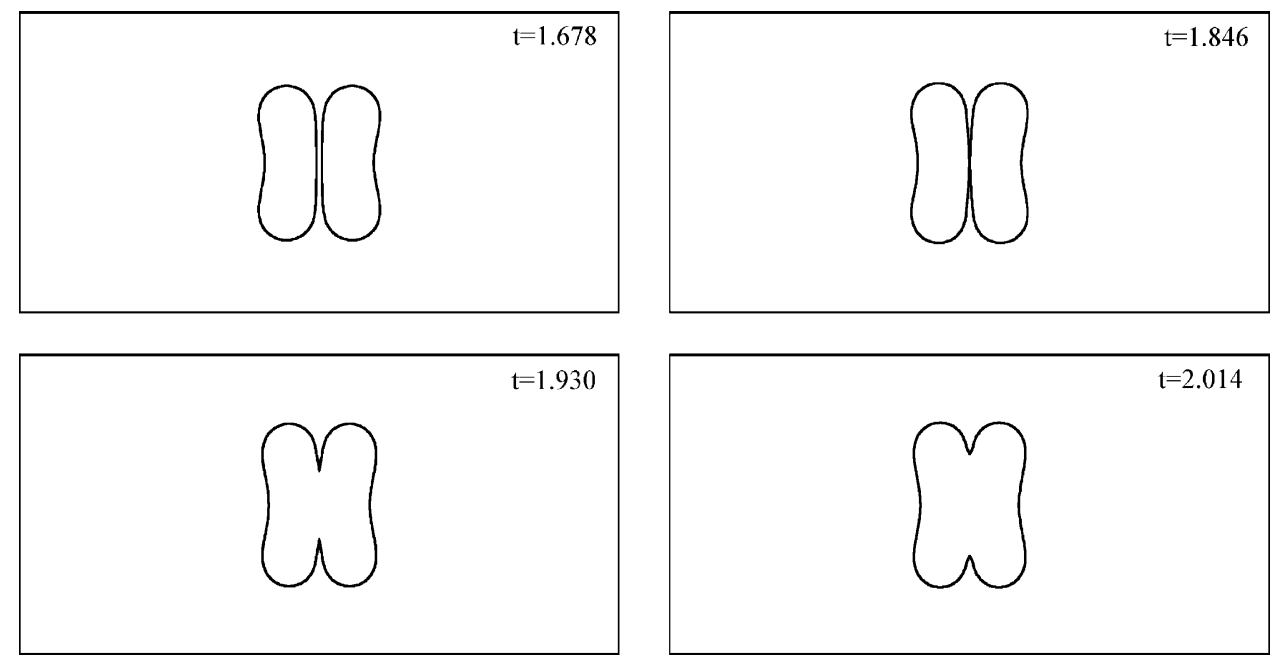

Fig. 4. Collision and coalescence of two Newtonian drops in a Newtonian matrix with a thicker interface. The parameters are the same as Fig. 1 except for $\epsilon=0.02$.

We have varied the degree of "initial deformation" by artificially using a higher surface tension (with the surface energy density $\lambda$ a hundred times larger) for various lengths of time before coalescence. An example is shown in Fig. 6 in which the higher interfacial tension, corresponding to $W e=0.12$, is applied for $t \leq 0.503$. Compared with Fig. 1, the initial deformation is smaller and the interfaces between the drops have a larger curvature. This results in a speedier film drainage and coalescence. Note also the lack of an inflection point on the interface between film rupture; no matrix fluid is entrapped in the resultant drop.

\subsection{Role of viscoelasticity in coalescence}

To investigate the role of viscoelasticity in film drainage, we compare three runs that are identical except for the rheology of the matrix or drop fluid: Newtonian drops in a Newto- nian matrix $(N / N)$, Newtonian drops in an Oldroyd-B matrix $(N / O)$ and Oldroyd-B drops in a Newtonian matrix $(O / N)$. The Oldroyd-B and Newtonian fluids have the same shear viscosity. This scheme is complicated somewhat by the fact that the coalescence process depends on the initial deformation of the drops. For two viscoelastic drops, the initial acceleration and approach will lead to a slightly different geometry at what may be considered the start of film drainage than for two Newtonian drops. To obtain a uniform initial drop shape, we place two Newtonian drops closer to each other at $d=1.2 D$ in a Newtonian matrix, accelerate them using a larger body force for a shorter period $t=0.087$, and suppress their initial deformation by temporarily raising the interfacial energy $\lambda$ by a factor of 100 . At the end of the acceleration, the original $\lambda$ is restored, and the phase function and velocity at $t=0.087$ are used as the "initial condition" for all the calculations in this section including $N / O$ and $O / N$ cases. For the viscoelas-
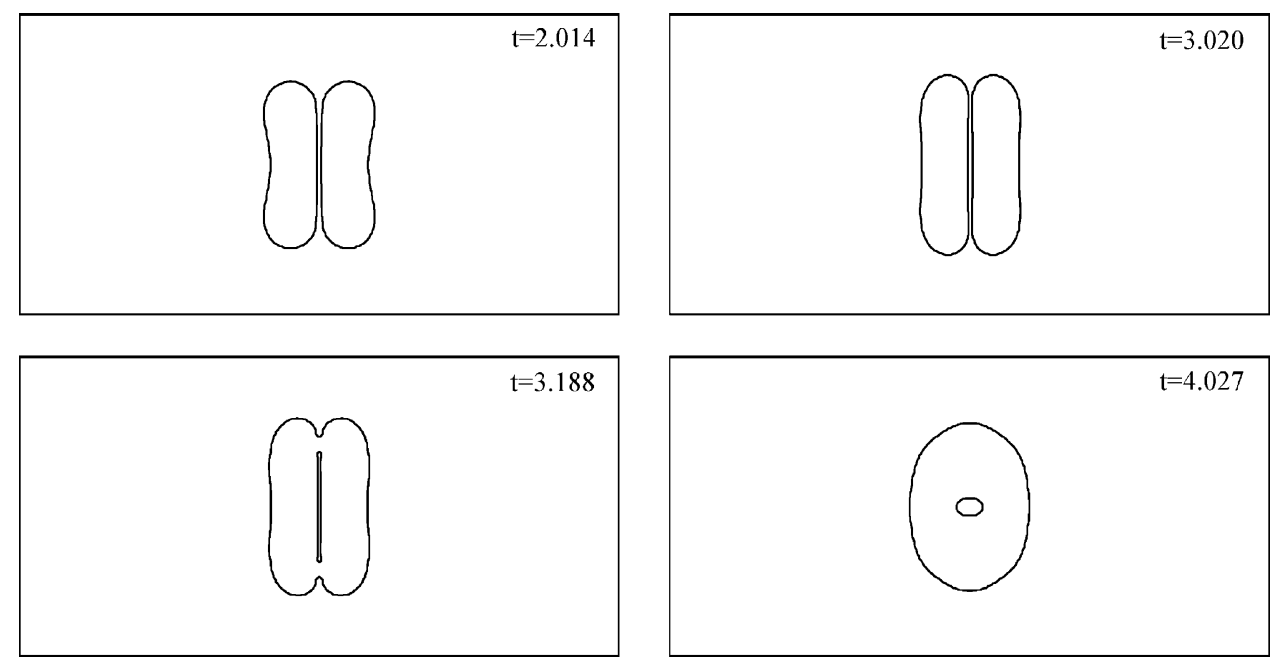

Fig. 5. Collision and coalescence of two Newtonian drops in a Newtonian matrix with a thinner interface. The parameters are the same as Fig. 1 except for $\epsilon=0.005$. 

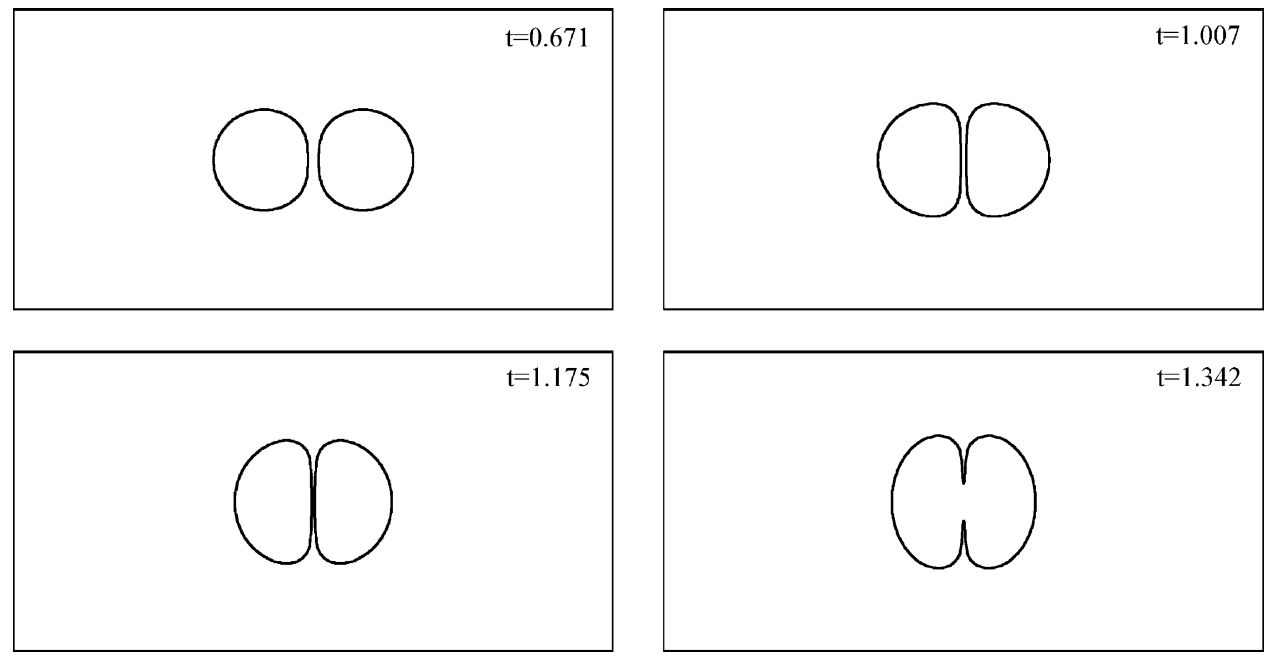

Fig. 6. Collision and coalescence of two Newtonian drops in a Newtonian matrix, with initial deformation artificially suppressed by using an interfacial energy 100 times larger until $t=0.503$. The Weber number is $W e=0.12$ before $t=0.503$ and $W e=12$ afterwards. Other parameters are the same as Fig. 1 .

tic fluid the polymer stress is simply set to zero at $t=0.087$. This gives us an identical "initial configuration" at $t=0.087$ for all three runs. The $N / N$ case is illustrated in Fig. 7. Note that the drops are essentially undeformed at $t=0.087$, with a minimum film thickness $h_{\min }=0.171$. During film drainage, the minimum thickness always occurs at the middle of the film, and no matrix filament is trapped in the combined drop.

Fig. 8 compares the thinning of the film thickness $h_{\min }$ for the three cases at several values of the Deborah number
$D e=U \lambda_{\mathrm{H}} / D$. When either the matrix or the drop phase is viscoelastic, the film drainage is faster than for $N / N$. Moreover, this effect becomes stronger with increasing Deborah number $D e$. This trend is also born out by the velocity profile of the drainage flow in various cases (Fig. 9).

Let us consider the $N / O$ case first where the matrix is an Oldroyd-B fluid. The flow in the draining film is essentially planar extension; this has been verified by calculating the components of the strain-rate tensor. Fig. 10 shows the
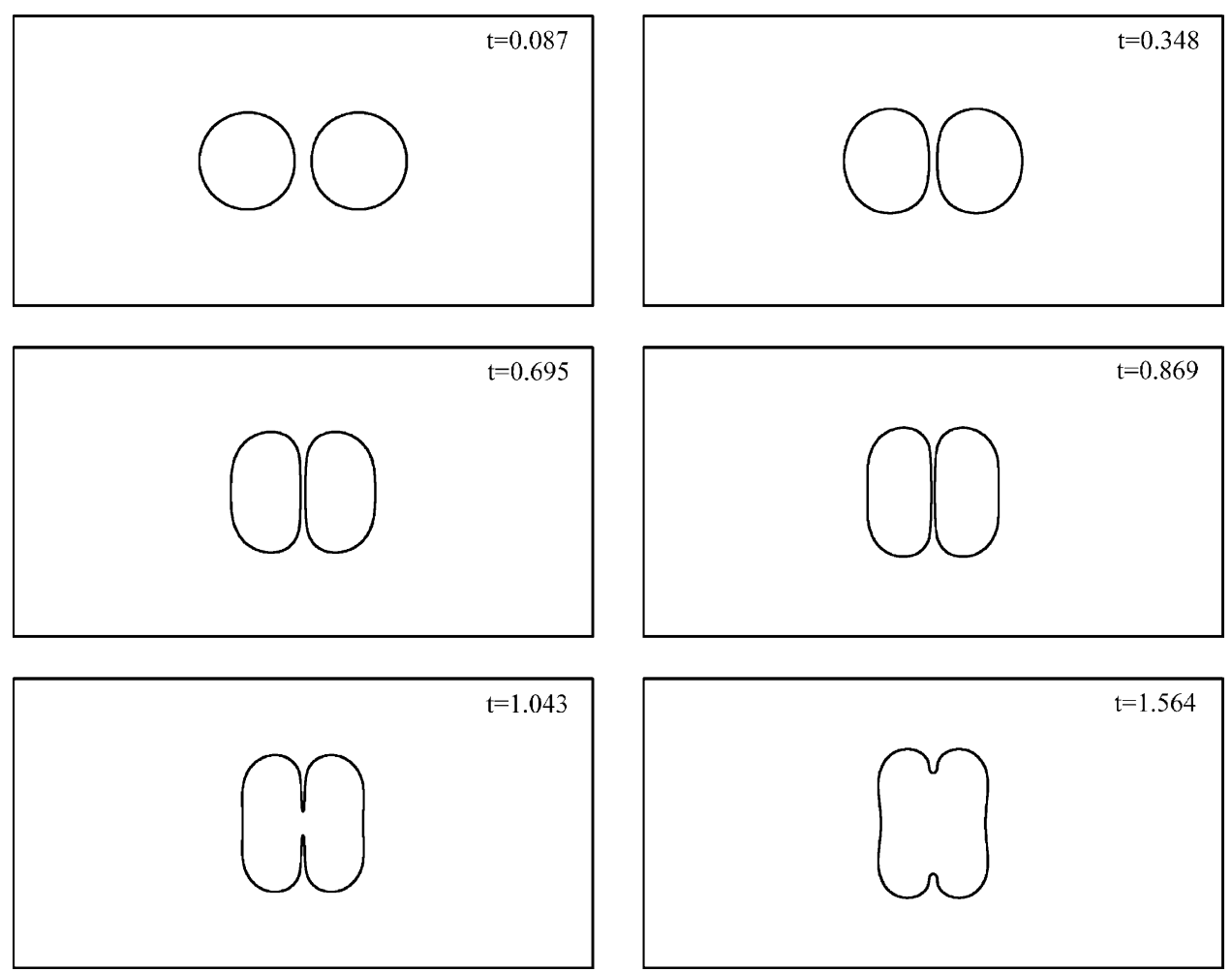

Fig. 7. Collision and coalescence of two Newtonian drops in a Newtonian matrix, with initial deformation artificially suppressed by using an interfacial energy 100 times larger until $t=0.087$. The Reynolds number is $\operatorname{Re}=26.1$, and the Weber number is $W e=9.6$. Other parameters are: $\epsilon=0.01$ and $\gamma=3.48 \times 10^{-5}$. 

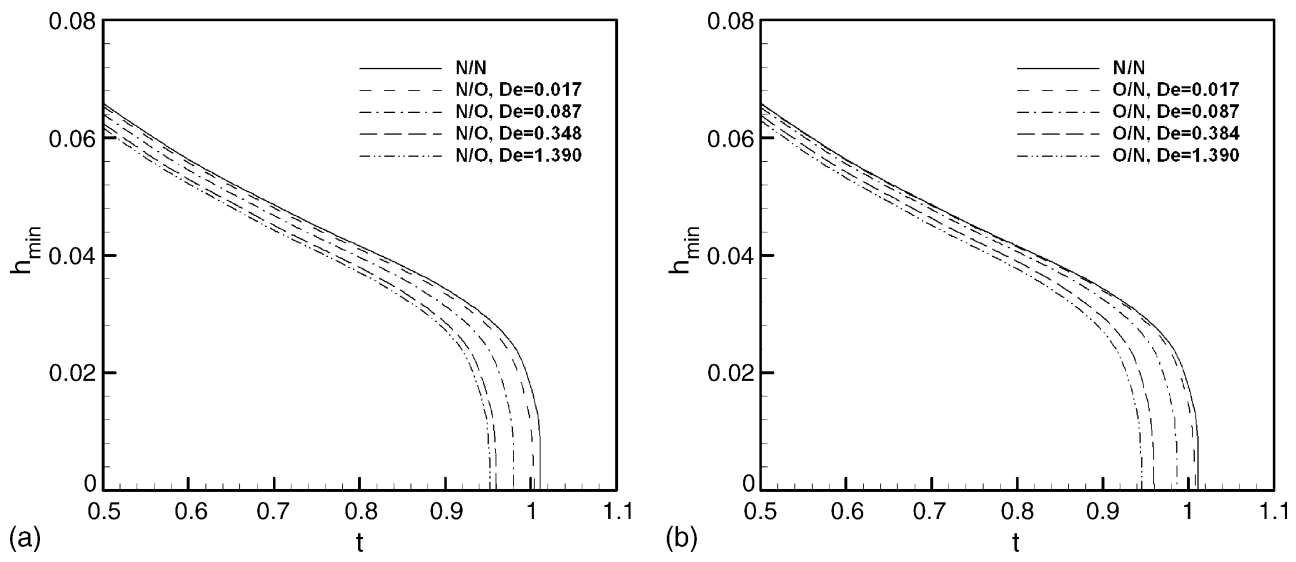

Fig. 8. Decrease of the minimum film thickness $h_{\min }$ during film drainage. (a) Comparison of $N / O$ runs at four Deborah numbers with the $N / N$ case; (b) comparison of $O / N$ runs at four Deborah numbers with the $N / N$ case. All other parameters are the same as in Fig. 7.
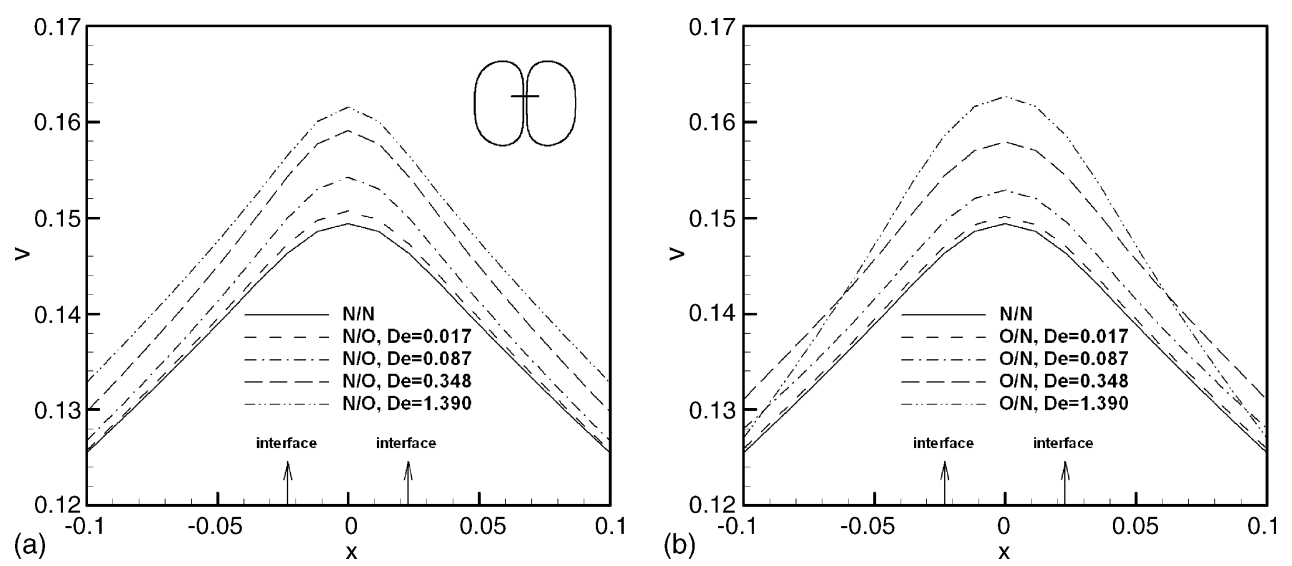

Fig. 9. Velocity profiles $v(x)$ along a horizontal line $y=0.105$ (position shown in the inset) for the draining flow in Fig. 8 at $t=0.695$. (a) Newtonian drops in an Oldroyd-B matrix $(N / O)$; (b) Oldroyd-B drops in a Newtonian matrix $(O / N)$. The $x$ and $y$ axes are along and normal to the direction of drop acceleration, with the origin at the center of the film.

temporal variation of the extensional rate $\dot{\epsilon}$ at the center of the film. While the drops accelerate toward each other, the strain rate must have increased. When the acceleration stops at $t=0.087$ and the film drainage commences, the strain rate starts to decrease in time. The upturn toward the end is due to short-range forces discussed before, and corresponds to the precipitous decrease of film thickness just before coalescence (Fig. 8). In this final stage, the disjoining pressure $\Pi_{\phi} \sim \lambda / \epsilon^{2}$ [cf. Eq. (14)] is much larger than the pressure drop caused by interfacial tension $\Delta p \sim \lambda / \epsilon D$ and dominates the film drainage process. Before this $(t<0.8$, say), the drainage can be approximately described by a power-law: $h_{\min } \propto t^{-0.8}$, which is somewhat faster than the Newtonian scaling $h_{\min } \propto t^{-2 / 3}$ predicted by lubrication theory for "partially mobile" interfaces[19]. In this power-law regime, our value of $h_{\min }$ is within $20 \%$ of the Newtonian prediction. Such a small discrepancy is unexpected since our calculations are in 2D with inertia, viscoelasticity and diffuse interfaces.

The reaction of the Oldroyd-B matrix to this timedependent squeezing flow is illustrated by the normal stress difference $N_{1}=\tau_{y y}-\tau_{x x}$ and elongational viscosity $\eta=$

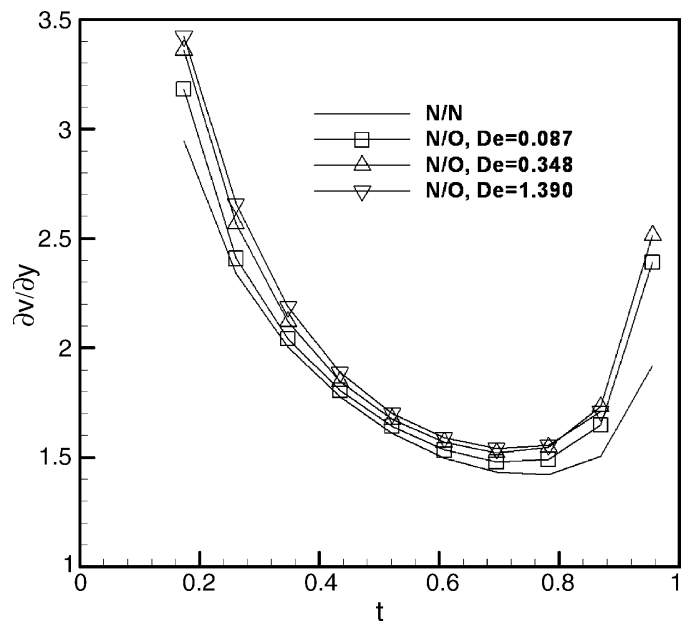

Fig. 10. Variation of the extension rate $\dot{\epsilon}=\partial v / \partial y=-\partial u / \partial x$ at the center of the film during drainage. The drops are Newtonian while the matrix is Newtonian or Oldroyd-B with various relaxation times. 

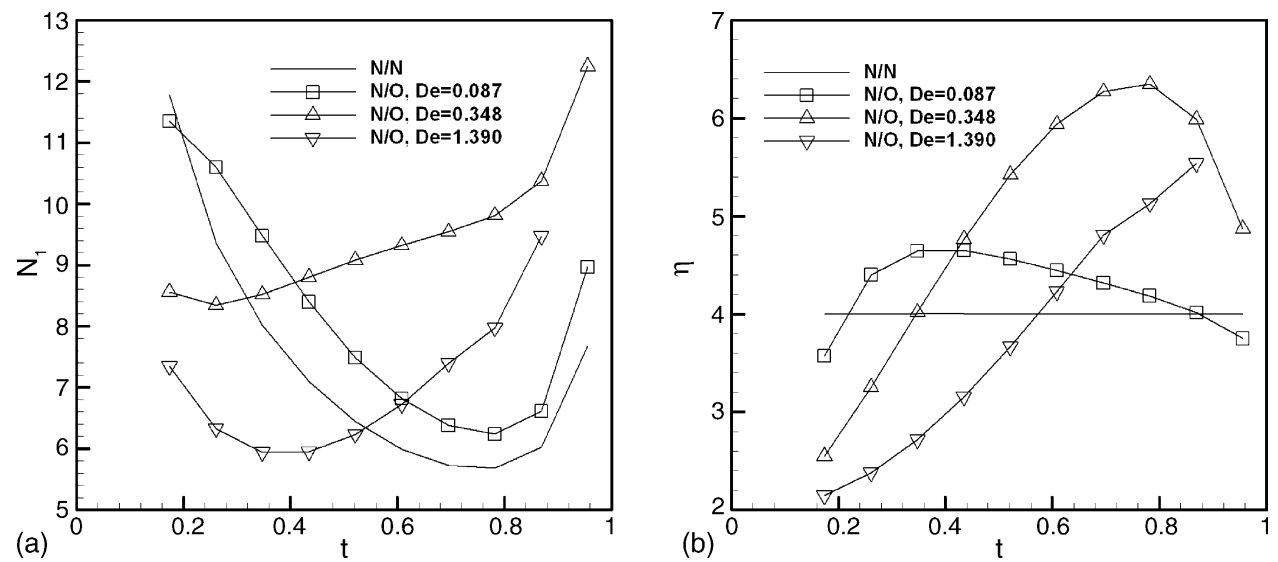

Fig. 11. Evolution of (a) the normal stress difference and (b) the elongational viscosity at the center of the film for the same simulations as in Fig. 10.

$N_{1} / \dot{\epsilon}$ in Fig. 11. The Newtonian fluid has a constant $\eta$, of course, and its normal stress $N_{1}$ traces the extension rate. The Oldroyd-B fluids have lower $N_{1}$ and $\eta$ at the beginning of the drainage process since the polymer chains are predominantly in the coiled state. They are stretched later by the extensional flow, and continue to unravel even as $\dot{\epsilon}$ declines. Hence, a cross-over occurs both in $N_{1}$ and in $\eta$, at a time $t_{c}$ that roughly scales with the polymer relaxation time. For $t<t_{c}$, the Oldroyd-B fluids present less elongational viscosity than the Newtonian fluid, which implies faster drainage. For $t>t_{c}$, on the other hand, the viscoelastic normal stress grows beyond that of the Newtonian stress. As a result, the Oldroyd-B fluids now exhibit a stronger resistance to deformation. The drainage is then hampered by viscoelasticity. This scenario is confirmed by Table 1, which lists the time $\Delta t$ for the final stage of drainage from a film thickness $h_{\min }=0.04-0$. With increasing $D e, \Delta t$ decreases first and then increases. $\Delta t$ for $N / O$ at $D e=0.017$ being slightly longer than that of $N / N$ is a numerical artifact due to errors in determining the exact time when $h_{\min }$ reaches 0.04 . Only at large enough De can the Oldroyd-B fluid attain a stress much higher than its Newtonian counterpart, and the hardening of the polymer is felt only toward the end of the drainage process. In fact, if we measure the time taken to drain the film from a larger initial thickness $h_{\min } \geq 0.06-0$, then the upturn in $\Delta t$ for large $D e$ will not appear. To summarize, the apparent viscoelastic effect of hastening film drainage and drop coalescence in Fig. 8 is actually the integrated result of two stages of opposing tendencies. The first stage, where the polymeric matrix offers weaker resistance to extension, dominates the second stage, where the high level of viscoelastic stress suppresses film drainage. Conceivably, for different parameter values or a different constitutive model, the trend may be reversed. We will return to this point shortly when comparing our simulations with experiments.

Essentially the same argument applies to Oldroyd-B drops coalescing in a Newtonian matrix $(O / N)$. Fig. 12 illustrates the evolution of the normal stress difference $N_{1}$ and the elongational viscosity $\eta$ just inside the Oldroyd-B drop (at $\phi=0.9$ ). The viscous normal stress from the baseline $N / N$ case is also shown for comparison. The viscoelastic stress is initially slow in developing but in time exceeds that of the Newtonian stress. This resembles Fig. 11 except that the viscoelastic stress now acts on the drop side of the interfaces. The effect on film drainage is the same, however, considering that the interfaces have negligible curvature over most of the film, and simply
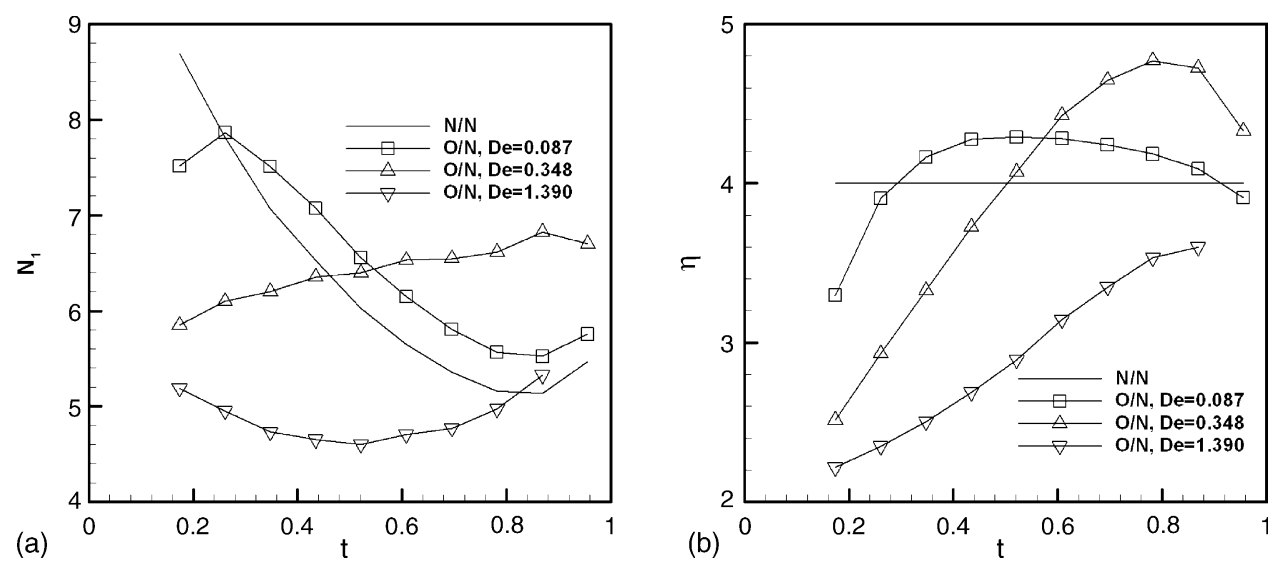

Fig. 12. Evolution of (a) the normal stress difference and (b) the elongational viscosity at the intersection of $y=0$ and $\phi=0.9$, just inside the Oldroyd-B drop. The matrix is Newtonian. 
Table 1

Duration of the final stage of film drainage as $h_{\min }$ decreases from 0.04 to 0

\begin{tabular}{ll}
\hline Test case & $\Delta t$ \\
\hline$N / N$ & 0.541 \\
$N / O, D e=0.017$ & 0.545 \\
$N / O, D e=0.087$ & 0.533 \\
$N / O, D e=0.348$ & 0.542 \\
$N / O, D e=1.390$ & 0.555 \\
$O / N, D e=0.017$ & 0.540 \\
$O / N, D e=0.087$ & 0.514 \\
$O / N, D e=0.348$ & 0.500 \\
$O / N, D e=1.390$ & 0.508
\end{tabular}

transmit the viscoelastic stress into the film. Finally, the duration of the last stage of drainage $\Delta t$ first decreases with $D e$ and then turns up, in a similar way to the $N / O$ case (Table 1).

Numerous experiments have been carried out on drop coalescence in Newtonian fluids, especially with surfactants on the interface [26-28]. In contrast, we have found only two experimental studies that used polymer solutions. Zdravkov et al. [19] measured the minimum film thickness as a function of time during coalescence between two viscoelastic drops in a Newtonian matrix. Perhaps because of adsorption of polymer molecules on the interface, the drops are to a large extent immobilized. Thus, $h_{\min }(t)$ obeys the Newtonian scaling for immobile film drainage, and the viscoelasticity in the drop component is not at all manifested. More interestingly, Dreher et al. [29] were able to investigate the effects of viscoelasticity in the matrix phase on the coalescence between a Newtonian drop and an essentially flat interface between the two components. For larger drops, viscoelasticity is found to reduce coalescence time. For smaller ones, on the other hand, film drainage is slower than in a comparable Newtonian matrix, and coalescence is delayed. By estimating the strain rate in the film for various drop sizes, Dreher et al. [29] argued that for smaller drops, the deformation is sufficiently strong to produce a large elastic stress that resists film drainage. For larger drops, the flow is weak so the matrix viscosity is below that of a Newtonian matrix having the same steady shear viscosity. Qualitatively, these are the same two scenarios that we have illustrated in Fig. 11 and Table 1.

\section{Drop retraction}

Drop retraction is a convenient method for measuring the interfacial tension between the drop and matrix fluids $[30,31]$. The basis of this measurement is the relationship between the evolution of the drop shape and the interfacial tension. Various phenomenological formulae have been developed by assuming Newtonian rheology in both the drop and the matrix phases, and they typically give similar results [32-34]. Maffettone and Minale's [32] model, for example, describes the retraction of an ellipsoidal drop by:

$L^{2}-B^{2}=\left(L^{2}-B^{2}\right)_{t=0} \exp \left[-\frac{\sigma}{\mu_{\mathrm{m}} R_{0}} f(\beta) t\right]$, where $R_{0}$ is the equilibrium drop radius, $\mu_{\mathrm{m}}$ is the matrix viscosity, $f(\beta)=40(\beta+1) /(2 \beta+3)(19 \beta+16)$ and $\beta$ is the viscosity ratio between the drop and the matrix. By measuring $L(t)$ and $B(t)$, the half-length and half-width of the drop, the interfacial tension $\sigma$ can be calculated from curve fitting. Since these models are intended for Newtonian fluids, it is surprising that they give fairly good results for some twophase polymeric systems [30].

The simulations in this section aim to elucidate the effects of viscoelasticity, in either the drop or the matrix phase, on drop retraction. One of the two components is Newtonian and the other is an Oldroyd-B fluid. The calculations are in $2 \mathrm{D}$, but we expect the physical insights gained here to be relevant to the $3 \mathrm{D}$ experiments. As in previous theoretical and numerical calculations, we assume the retraction is slow and the fluids are highly viscous so as to render inertia negligible. For simplicity, the two components are assumed to have the same density and same steady-shear viscosity. The geometry of the problem is shown in Fig. 13. Initially, the drop is elliptic with semi-axes $L_{0}$ and $B_{0}$. We will use the final drop radius $R_{0}=\sqrt{L_{0} B_{0}}$ as the characteristic length.

Let us first consider the retraction of an elliptic drop from a stationary initial state with zero velocity and zero stress. This is intended to mimic experiments where the elongated drop is produced by melting a filament sandwiched between two sheets of the matrix fluid [30]. Depending on how the filament is produced, there may be residual stress frozen inside, but we neglect this possibility. For most results in this section the initial geometric parameters are $W=H=6, L_{0}=1.1055$ and $B_{0}=0.9045$, which correspond to an initial deformation parameter $D_{0}=\left(L_{0}-B_{0}\right) /\left(L_{0}+B_{0}\right)=0.1$. From the equilibrium drop radius $R_{0}$, viscosity $\mu$ and interfacial tension $\sigma$, we define a characteristic time scale for retraction:

$t_{\text {ret }}=\frac{\mu R_{0}}{\sigma}$.

The Deborah number is defined as $D e=\lambda_{\mathrm{H}} / t_{\mathrm{ret}}$, where $\lambda_{\mathrm{H}}$ is the relaxation time of the polymer. Note that $D e$ differs

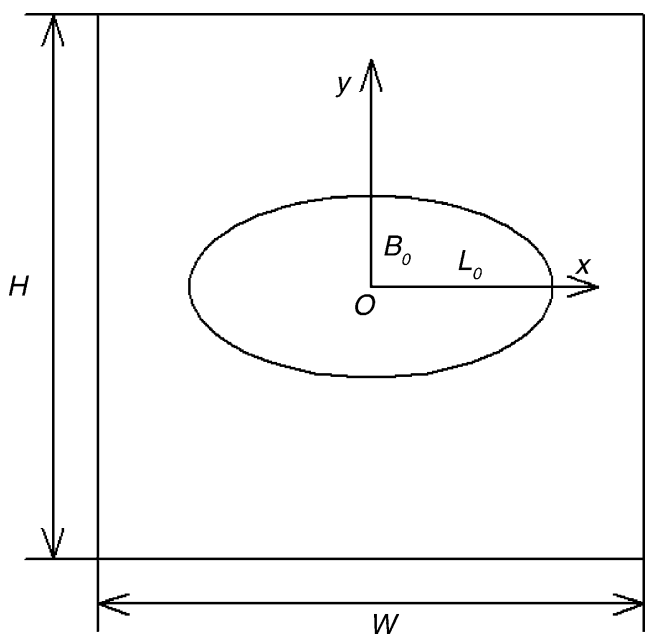

Fig. 13. Computational domain for drop retraction. 


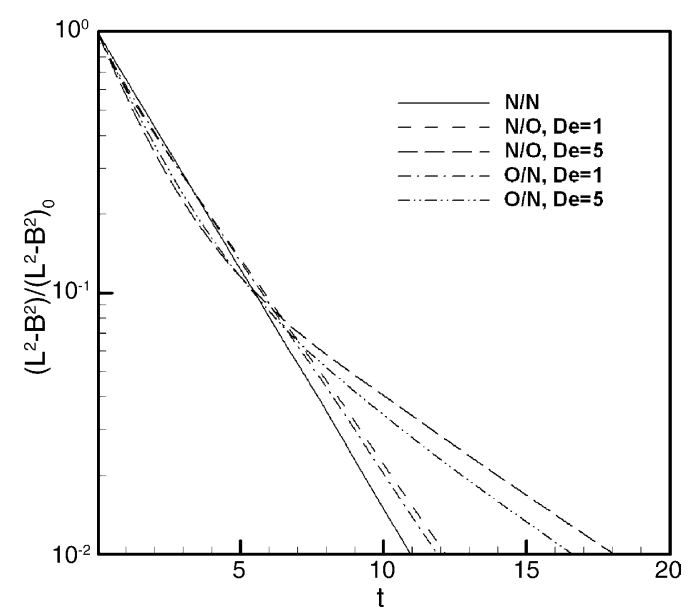

Fig. 14. The $L^{2}-B^{2} \sim t$ curves for drop relaxation from an initially elliptic shape with $D_{0}=0.1$.

from its usual definition using a strain rate by a factor that is the capillary number. Two Deborah numbers are simulated: $D e=1$ and 5. The other dimensionless parameters are: $\epsilon=$ $0.01, \lambda=1.0607 \times 10^{-2}$ (corresponding to $\sigma=1$ ). For the Oldroyd-B fluid, the retardation time is equal to the relaxation time.

Fig. 14 compares several cases of drop retraction, with a Newtonian drop in an Oldroyd-B matrix $(\mathrm{N} / \mathrm{O})$, an Oldroyd-B drop in a Newtonian matrix $(O / N)$, and a Newtonian drop in a Newtonian matrix $(N / N)$ as the baseline. Perhaps surprisingly, the $O / N$ and $N / O$ cases differ very little for the same $D e$. This is because the interfacial tension drives the retraction against resistance from both the drop and the matrix, and whether the viscoelasticity occurs in the drop or the matrix, it produces roughly the same amount of resistance. First, the fluids in the drop and in the matrix are subject to roughly equal extension rates, but with opposite signs. At the pointed ends of the drop, for instance, the interface compresses the drop fluid and stretches the matrix fluid, regardless of their rheology. This can be seen from the velocity profile $u(x)$ in Fig. $15 ;|u|$ reaches its maximum near the interface (at $x \approx 1.02$ ) for both $N / O$ and $O / N$. Furthermore, the velocity gradient $\partial u / \partial x$ - or the extension rate- has roughly the same magnitude on both sides of the interface and between $N / O$ and $O / N$. A similar argument can be made at the "waist" of the drop, i.e., the ends of the minor axes.

Second, we may write out the polymer tensile stress for a planar extensional flow $(u, v)=(\dot{\epsilon} x,-\dot{\epsilon} y)$ of an Oldroyd-B fluid [35]:

$\tau_{p, x x}=\frac{2 \mu_{p} \dot{\epsilon}}{1-2 \lambda_{\mathrm{H}} \dot{\epsilon}}\left\{1-\exp \left[-\frac{t}{\lambda_{\mathrm{H}}}\left(1-2 \lambda_{\mathrm{H}} \dot{\epsilon}\right)\right]\right\}$.

Given the small value of $\partial u / \partial x$ at the interface, the local Deborah number $\left|\lambda_{H} \dot{\epsilon}\right| \ll 1$. Eq. (20) implies that the polymers will produce roughly the same amount of stress if stretched or compressed at the same $|\dot{\epsilon}|$. In other words, between $N / O$ and $O / N$, the polymer normal stress $\tau_{p, x x}$ has more or less the same magnitude on the polymer side, as does the vis-

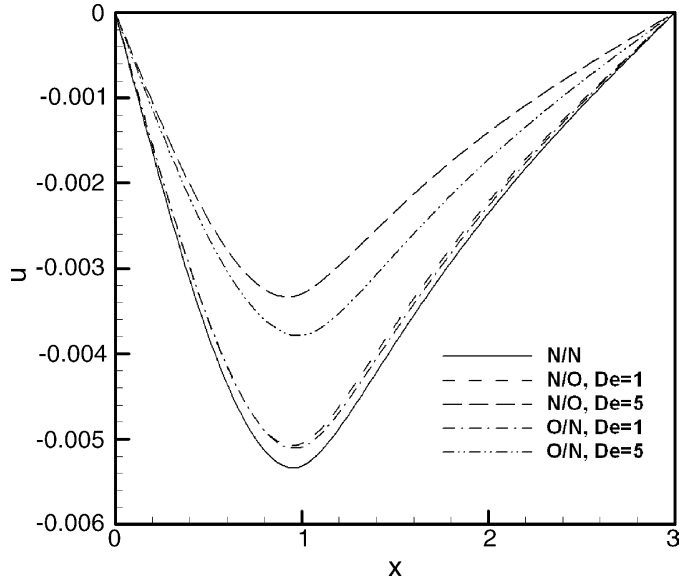

Fig. 15. Local velocity profiles $u(x)$ across the interface at $t=5$. The interface is at $x \approx 1.02$.

cous normal stress on the Newtonian side. Therefore, the total resistance to retraction is roughly the same for the two cases. Hence, the proximity of $O / N$ and $N / O$ curves in Fig. 14. This contrasts flow-induced drop deformation (e.g., [36,37]) where the two cases present opposite trends. There, the deformation is driven by a prescribed flow and the interplay between interfacial tension and normal stresses is different.

The second notable feature is the effect of $D e$, which can be explained as in Section 3.2. Owing to the finite time needed for stress growth in a viscoelastic fluid, the resistance to retraction is initially weaker than in a Newtonian fluid. Hence, the retraction is faster in the initial stage for $N / O$ and $O / N$ than for $N / N$. This is reversed later as the viscoelastic stress exceeds the Newtonian stress, and the retraction becomes slower than that for $N / N$. Not surprisingly, this effect is more pronounced at higher $\mathrm{De}$.

Based on the above discussion, the drop retraction method for measuring interfacial tension is likely to work for viscoelastic fluids if the retraction is slow (due to high viscosity, say), and the Deborah number is small. Otherwise the retraction does not follow an exponential law and the method will fail. Indeed, the experiments of Mo et al. [30] and Son and Yoon [31] involve retraction times of many hundreds of seconds. Assuming a polymer relaxation time of several seconds, the Deborah number as defined here will be $D e \sim 0.01$.

Most previous work on drop retraction deals with a different initial condition: the cessation of a steady shear or elongational flow that has deformed the drop to a steady shape. Compared with the retraction discussed above, a new factor is the initial stress field; the polymer chains are stretched and their relaxation will be coupled with the relaxation of the drop shape. We have simulated drop retraction from initial conditions produced by steady shear at a capillary number of $C a=0.1$; Fig. 16 compares the behavior of $O / N, N / O$ and $N / N$ systems. Note that the initial deformation $D_{0}$ differs slightly among the five runs because of the different viscoelasticity of the components. It is also slightly larger than that in Fig. 14. 


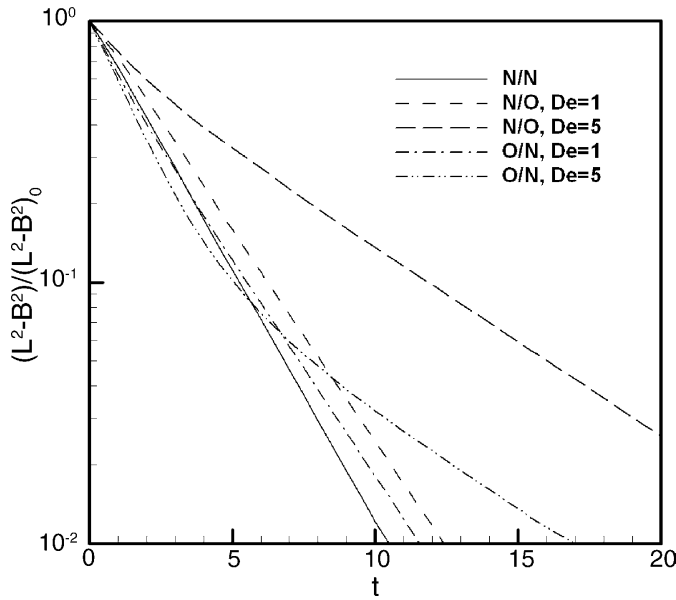

Fig. 16. The $L^{2}-B^{2} \sim t$ curves for drop relaxation from initial conditions produced by steady shear. For the 5 runs, the initial deformation parameters are $D_{0}=0.1078(N / N), 0.1065(N / O, D e=1), 0.1035(N / O, D e=5)$, $0.1072(O / N, D e=1)$, and $0.1053(O / N, D e=5)$. The computational domain is $8 \pi \times 8$, somewhat larger than for Fig. 14 .

Despite the differing $D_{0}$, the retraction of an Oldroyd-B drop in a Newtonian matrix $(O / N)$ is very close to that starting with zero initial stress (Fig. 14). In contrast, the retraction of a Newtonian drop in an Oldroyd-B matrix $(N / O)$ is quite different from its counterpart in Fig. 14. The retraction is slower than the $N / N$ case from the very beginning, and the discrepancy grows with time. The effect is also more pronounced for higher $D e$. These observations can be explained by studying the flow field and the polymer stress distribution. During the steady shear prior to retraction, the flow inside the drop is highly rotational in all cases, while outside it is shear or moderately extensional (Fig. 17). Thus, if the matrix is viscoelastic, a considerable normal stress builds up around the drop. In contrast, if the drop is viscoelastic, there is little polymer stress inside the drop. Fig. 18 plots the evolution of the normal stress difference $N_{1}=\tau_{n n}-\tau_{t t}$ just outside the

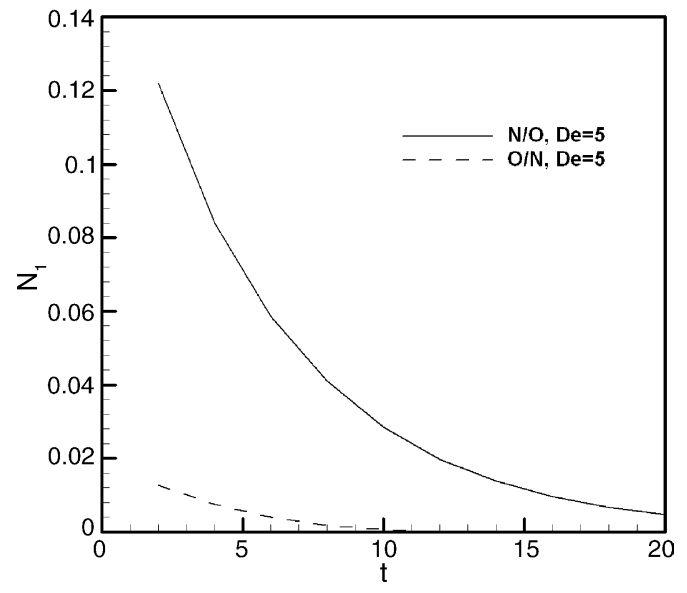

Fig. 18. Normal stress difference in the Oldroyd-B fluid near the drop tip. The data are take at the intersection of $\phi=0.9$ and the major axis of drop. Recall that $\phi=1$ in the bulk Oldroyd-B fluid.

end of the drop for $N / O$ and just inside for $O / N$ during retraction, $n$ and $t$ being the local normal and tangential direction. The former is much larger and lasts longer, and explains the retarded retraction in the Oldroyd-B matrix. The persistence of polymer stress outside the contracting ends of the drop is partly due to the fact the retraction further stretches the polymer chains or at least postpones their recoiling [38]. This effect is significant since $D e=\lambda_{\mathrm{H}} / t_{\mathrm{ret}} \geq 1$, with the polymer chain relaxing more slowly than the drop. Also note that for $O / N$, the small residual viscoelastic stress inside the drop tends to promote drop retraction initially because $N_{1}>0$.

The experiment most relevant to our simulation is that of Tretheway and Leal [38], who measured the retraction of Newtonian drops suspended in a PIB/PB Boger fluid following planar extensional flow. Although quantitative comparison is precluded by the different flow types and dimensionality, the qualitative trends are the same. In particular, Tretheway and Leal [38] suggested that the tensile
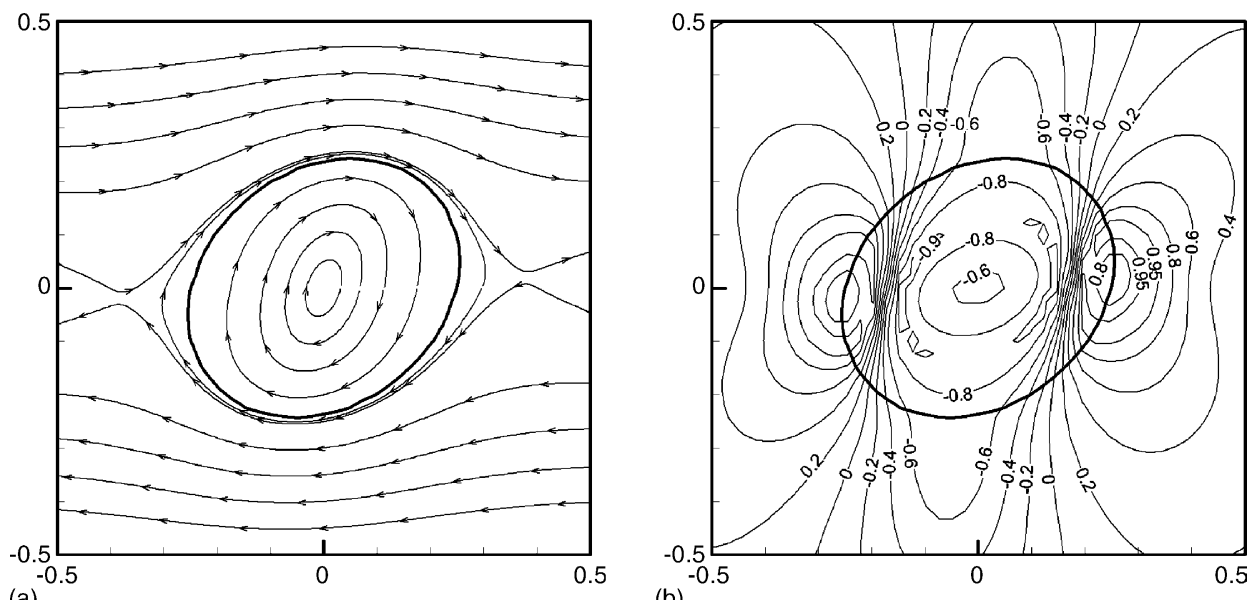

Fig. 17. Streamlines (a) and flow type contours (b) near a drop in steady shear. Both the drop and the matrix are Newtonian. $\mathrm{Ca}=0.1$. The flow type parameter is defined as $\alpha=\frac{\|\boldsymbol{D}\|-\|\boldsymbol{\Omega}\|}{\|\boldsymbol{D}\|+\|\boldsymbol{\Omega}\|}$, where $\boldsymbol{D}=\left(\nabla \boldsymbol{v}+(\nabla \boldsymbol{v})^{\mathrm{T}}\right) / 2, \boldsymbol{\Omega}=\left((\nabla \boldsymbol{v})^{\mathrm{T}}-\nabla \boldsymbol{v}\right) / 2$, and the norm of the matrices is defined by, e.g., $\|\boldsymbol{D}\|=\sqrt{\frac{\boldsymbol{D}: \boldsymbol{D}^{\mathrm{T}}}{2}}$. 
stress induced by the contraction of the drop ends causes the drop retraction to slow down in a viscoelastic matrix. This is essentially borne out by our Figs. 16 and 18. In addition, the prediction that persisting polymer stresses in the matrix hinder drop retraction confirms similar predictions by the phenomenological models of Maffettone and Greco [39] and Yu et al. [40]. Hooper et al. [41] computed the retraction of a drop pre-deformed by a uniaxial elongational flow. The cessation of flow, however, is effected by instantaneously freezing the outer boundaries of the computational domain. The induced backflow inside the domain causes the drop to stretch further before retraction.

\section{Summary}

In this paper, we applied a diffuse-interface formulation to drop coalescence and retraction involving Newtonian and Oldroyd-B fluids. Somewhat coincidentally, in most cases the viscoelasticity works in much the same way whether it occurs in the drop or in the matrix. The only exception is drop retraction after cessation of steady shear.

The diffuse interface makes it possible to simulate topological changes such as drop coalescence. The final stage of the coalescence is dominated by short-range molecular forces, and we have shown that the Cahn-Hilliard energy produces such a force comparable with the van der Waals force. Viscoelasticity in either component hastens film drainage and drop coalescence, and the effect can be explained by the relatively slow stress growth in the non-Newtonian component. Drop retraction is generally hindered by viscoelasticity, owing to the persistence of the polymer stresses. As a result, one cannot infer the interfacial tension in general from the retraction process using Newtonian models.

Within the theoretical framework of the current paper, the above results have been rationalized by analyzing the flow and stress fields near the interfaces. On the other hand, direct comparison with experiments is hampered by the two-dimensionality of our simulations and the scarcity of experimental data, especially for the late stage of coalescence. Nevertheless, the physical insights gained here on viscoelastic effects during drop coalescence and retraction are consistent with, and capable of qualitatively explaining, prior experimental observations. Such insights are particularly valuable for the film rupture process, which is difficult to document experimentally owing to the short-range forces and singular topological changes.

\section{Acknowledgments}

Acknowledgment is made to the Donors of The Petroleum Research Fund, administered by the American Chemical Society, for partial support of this research. J.J.F. was also supported by the NSF (CTS-0229298, CTS-9984402), the NSERC and the Canada Research Chairs program, and the
NNSF of China (No. 20174024 and No. 20490220). J.S. was supported by the NSF (DMS-0074283, DMS-0311915). C.L. was supported by the NSF (DMS-0405850). We acknowledge discussions with Professor M. M. Denn, Professor W. Yu and Professor C. Zhou.

\section{References}

[1] S. Guido, F. Greco, Dynamics of a liquid drop in a flowing immiscible liquid, in: D.M. Binding, K. Walters (Eds.), Rheology Reviews 2004, The British Society of Rheology, 2004.

[2] L.A. Utracki, Polymer Alloys and Blends, Hanser, Munich, 1990.

[3] J.L. West, Polymer-dispersed liquid crystals, in: R.A., Weiss, C.K., Ober (Eds.), Liquid-Crystalline Polymers, vol. 435, ACS Symp. Ser., ACS, Washington, D.C., 1990, pp. 475-495 (Chapter 32).

[4] L. Dintenfass, Blood as a near-'ideal' emulsion: a retrospective on the concept of the red cell as a fluid drop, its implications for the structure of the red cell membrane, Biorheology 27 (1990) 149-161.

[5] H.A. Stone, Dynamics of drop deformation and breakup in viscous fluids, Ann. Rev. Fluid Mech. 26 (1994) 65-102.

[6] J. Feng, G. Sgalari, L.G. Leal, A theory for flowing nematic polymers with orientational distortion, J. Rheol. 44 (2000) 1085-1101.

[7] J.A. Sethian, P. Smereka, Level set methods for fluid interfaces, Ann. Rev. Fluid Mech. 35 (2003) 341-372.

[8] T.E. Tezduyar, Computation of moving boundaries and interfaces and stabilization parameters, Int. J. Num. Methods Fluids 43 (2003) 555575.

[9] P. Yue, J.J. Feng, C. Liu, J. Shen, A diffuse-interface method for simulating two-phase flows of complex fluids, J. Fluid Mech. 515 (2004) 293-317.

[10] D.M. Anderson, G.B. McFadden, A.A. Wheeler, Diffuse-interface methods in fluid mechanics, Ann. Rev. Fluid Mech. 30 (1998) 139165.

[11] C. Liu, J. Shen, A phase field model for the mixture of two incompressible fluids and its approximation by a Fourier-spectral method, Physica D 179 (2003) 211-228.

[12] J. Lowengrub, L. Truskinovsky, Quasi-incompressible Cahn-Hilliard fluids and topological transitions, Proc. R. Soc. Lond. A 454 (1998) 2617-2654.

[13] C.S. Peskin, A random-walk interpretation of the incompressible navier-stokes equations, Commun. Pure Appl. Math. 38 (1985) 845852.

[14] Y. Gliklikh, Global Analysis in Mathematical Physics: Geometric and Stochastic Methods, Springer, New York, 1997.

[15] R.B. Bird, D.F. Curtiss, R.C. Armstrong, O. Hassager, Dynamics of Polymeric Liquids, vol. 2, Kinetic Theory, Wiley, New York, 1987.

[16] D. Jacqmin, Calculation of two-phase Navier-Stokes flows using phasefield modelling, J. Comput. Phys. 155 (1999) 96-127.

[17] J. Shen, Efficient spectral-Galerkin method. II. direct solvers of second and fourth order equations by using Chebyshev polynomials, SIAM J. Sci. Comput. 16 (1995) 74-87.

[18] M.R. Nobari, Y.-J. Jan, G. Tryggvason, Head-on collision of drops-a numerical investigation, Phys. Fluids 8 (1996) 29-42.

[19] A.N. Zdravkov, G.W.M. Peters, H.E.H. Meijer, Film drainage between two captive drops: PEO-water in silicon oil, J. Colloid Interface Sci. 266 (2003) 195-201.

[20] S.G. Bradley, C.D. Stow, Collision between liquid drops, Philos. Trans. R. Soc. Lond. Ser. A 287 (1978) 635-675.

[21] A. Bhakta, E. Ruckenstein, Decay of standing foams: drainage, coalescence and collapse, Adv. Colloid Interface Sci. 70 (1997) 1-124.

[22] M.B. Nemer, X. Chen, D.H. Papadopoulos, J. Blawzdziewicz, M. Loewenberg, Hindered and enhanced coalescence of drops in Stokes flows, Phys. Rev. Lett. 92 (2004) 114501.

[23] J.N. Israelachvili, Intermolecular and Surface Forces, Academic, New York, 1992. 
[24] L.M. Pismen, Nonlocal diffuse interface theory of thin films and the moving contact line, Phys. Rev. E 64 (2001) 021603.

[25] X. Yang, J. J. Feng, C. Liu, J. Shen, Numerical simulations of jet pinching-off and drop formation using an energetic variational phasefield method, J. Comput. Phys., 2005, submitted for publication.

[26] L.G. Leal, Flow induced coalescence of drops in a viscous fluid, Phys. Fluids 16 (2004) 1833-1851.

[27] J.W. Ha, Y. Yoon, L.G. Leal, The effect of compatibilizer on the coalescence of two drops in flow, Phys. Fluids 15 (2003) 849-867.

[28] D. Steven, Hudson, M. Alex, Jamieson, E. Brian, Burkhart, The effect of surfactant on the efficiency of shear-induced drop coalescence, J. Colloid Interface Sci. 265 (2003) 409-421.

[29] T.M. Dreher, J. Glass, A.J. O'Connor, G.W. Stevens, Effect of rheology on coalescence rates and emulsion stability, AIChE J. 45 (1999) 11821190.

[30] H. Mo, C. Zhou, W. Yu, A new method to determine interfacial tension from the retraction of ellipsoidal drops, J. Non-Newtonian Fluid Mech. 91 (2000) 221-232.

[31] Y. Son, J.T. Yoon, Measurement of interfacial tension by a deformed drop retraction method, Polymer 42 (2001) 7209-7213.

[32] P.L. Maffettone, M. Minale, Equation of change for ellipsoidal drops in viscous flow, J. Non-Newtonian Fluid Mech. 78 (1998) 227-241.
[33] N.E. Jackson, C.L. Tucker, A model for large deformation of an ellipsoid droplet with interfacial tension, J. Rheol. 47 (2003) 659-682.

[34] W. Yu, M. Bousmina, Ellipsoidal model for droplet deformation in Newtonian systems, J. Rheol. 47 (2003) 1011-1040.

[35] R.B. Bird, R.C. Armstrong, O. Hassager, Dynamics of Polymeric Liquids, vol. 1, Fluid Mechanics, Wiley, New York, 1987.

[36] F. Mighri, A. Ajji, P.J. Carreau, Influence of elastic properties on drop deformation in elongational flow, J. Rheol. 41 (1997) 1183-1201.

[37] F. Mighri, P.J. Carreau, A. Ajji, Influence of elastic properties on drop deformation and breakup in shear flow, J. Rheol. 42 (1998) 14771490.

[38] D.C. Tretheway, L.G. Leal, Deformation and relaxation of Newtonian drops in planar extensional flows of a Boger fluid, J. Non-Newtonian Fluid Mech. 99 (2001) 81-108.

[39] P.L. Maffettone, F. Greco, Ellipsoidal drop model for single drop dynamics with non-Newtonian fluids, J. Rheol. 48 (2004) 83-100.

[40] W. Yu, C. Zhou, M. Bousmina, Theory of morphology evolution in mixtures of viscoelastic immiscible components, J. Rheol. 49 (2005) 215-236.

[41] R.W. Hooper, V.F. de Almeida, C.W. Macosko, J.J. Derby, Transient polymeric drop extension and retraction in uniaxial extensional flows, J. Non-Newtonian Fluid Mech. 98 (2001) 141-168. 